\title{
Comparison of Different Methods for Determination of Delamination Initiation Point in the DCB Test on Coupled CFRP Composite Laminates
}

\author{
Jakub Rzeczkowski ${ }^{1,}$, , Sylwester Samborski ${ }^{1, b^{*}}$ \\ ${ }^{1}$ Lublin University of Technology, Department of Applied Mechanics, Nadbystrzycka 36 St, \\ Lublin 20-618, Poland \\ akubarzeczkowski@op.pl, bs.samborski@pollub.pl
}

\begin{abstract}
Keywords: Damage, Delamination, Double Cantilever Beam, Acoustic Emission, Mechanical Coupling
\end{abstract}

\begin{abstract}
This article presents an experimental determination of the mode I critical strain energy release rate (c-SERR) for different initiation definitions. The multidirectional (MD) and mechanically coupled laminates were subjected to the double cantilever beam (DCB) test. The $N L, P_{\max }$ and $5 \%$ criteria were taken into account in calculation of the fracture toughness $\left(G_{\mathrm{IC}}\right)$. An acoustic emission (AE) technique was used as additional initiation criterion. On the basis of the performed test it was found that the critical strain energy release rate depends on the adopted definition as well as on the specimen interface and mechanical couplings.
\end{abstract}

\section{Introduction}

The fiber reinforced plastic (FRP) laminates are widely used in many industry sectors. The main advantages of polymeric composites are high strength and corrosion resistance. Those materials are used in the manufacturing of the contemporary load carrying structures in airplanes, cars and marine industry [1]. During the operation, on the construction parts different and often variable loads act, as well as various environmental factors must be considered which can cause damage. The most common type of failure in the FRP laminates is called delamination which is the major weaknesses of advanced composite structures. Knowledge of a laminated composite materials resistance to interlaminar fracture is useful for product development and material section. The fracture toughness $\left(G_{\text {IC }}\right)$ in the form of the critical strain energy release rate (c-SERR) values can be determined for the mode I fracture in the experiment on Double Cantilever Beam (DCB). Such tests are standardized by the ASTM organization (see the ASTM D5528 Standard [2]), where different calculation methods are described. In the available literature, there are some papers addressing to delamination resistance problem for the FRP laminates taking into account the influence of fiber misorientation at delamination interface [3-4], as well as the effect of the mechanical coupling [5-12] on the critical strain energy release rate (c-SERR). One of the problems with precise determination of the mode I c-SERR may be fiber bridging phenomena. This mechanism results from growing the delamination between plies of dissimilar orientation in multiply laminated composite structures. Moreover, in the nonunidirectional DCB specimens a pure mode I fracture may not be achieved as a result of the mechanical couplings $[5,8,9]$. Another issue in computation of the $G_{\text {IC }}$ is properly determination of the initiation point. In the experiments led by the authors of the current paper, the difficulties with precise determination of delamination onset point where experienced. The knowledge of the actual value of the load corresponding to delamination onset is however of particular importance while computing the mode I fracture toughness in accordance with the respective ASTM Standard. Any uncertainty in experimental reading of the fracture onset load can lead to generating the inaccurate results in 
the $G_{\text {IC }}$ calculations. Therefore, four different definitions of initiations were used by the authors to calculate the critical strain energy release rate with the experimental results: the peak force $\left(P_{\max }\right)$, the deviation from the linearity point force $(N L)$, the $5 \%$ compliance increase force, as well as the acoustic emission (AE) indicated force. To obtained mode I c-SERR are the results of two calculation methods: the Modified Beam Theory (MBT) and the Compliance Calibration Method (CCM). The experimental results for the four different approaches of determination of the peak load are discussed in this paper.

\section{Test specimens}

Experimental DCB tests was performed on rectangular uniform thickness carbon epoxy composite laminates. The geometrical dimensions of the manufactured specimens was width $b=25 \mathrm{~mm}$, thickness $h=5 \mathrm{~mm}$ and total length equal $175 \mathrm{~mm}$. The composite samples contained nonadhesive insert on the mid plane that serves as a delamination initiator. The scheme and geometrical dimensions of the DCB specimens was presented in Fig. 1.

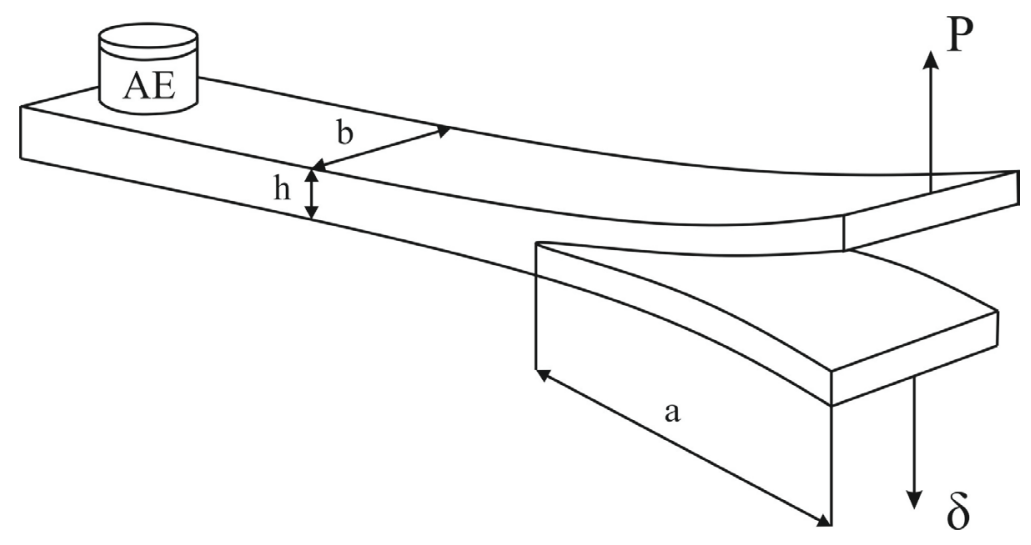

Fig. 1. DCB deformed specimen with AE sensor.

During the tests the multidirectional laminates with delamination interfaces $\left[30^{\circ} / 30^{\circ}\right]$ and [45\% $/ 45^{\circ}$ ] were used. Additionally, bending-twisting (BT) coupled laminate with the respective ply sequence: [45\% $\left./ 0^{\circ} / 45^{\circ} / 45^{\circ} / 0^{\circ} /-45^{\circ} / 0^{\circ} /-45^{\circ} /-45^{\circ} /-45^{\circ} /-45^{\circ} / 0^{\circ} /-45^{\circ} / 45^{\circ} / 0^{\circ} / 0^{\circ} / 45^{\circ} / 45^{\circ}\right]$ and bending-extension (BE) coupled laminate $\left[45^{\circ} /-45^{\circ} / 0^{\circ} /-45^{\circ} / 0^{\circ}\right.$ 45\% $\left./ 90^{\circ} / 45^{\circ} /-45^{\circ}\right]$ were examined.

\section{Experiment}

The experiment was performed on the Shimadzu ASG-X tensile testing machine with 5kN load cell according to the ASTM D5528 Standard indications. The samples with sticked piano hinges was mounted between the grips of the loading machine and then aligned and centered. Prior to test, both edges of the specimens was coated white fluid to aid in visual detection of delamination onset. The test was carried out with constant crosshead speed equal $1 \mathrm{~mm} / \mathrm{min}$ which caused quasi-static opening the specimens. During the experiment the applied load $P$ and displacement $\delta$ was measured and registered by the trapezium-X software. Delamination onset as well as all propagation values were visually observed and marked on the specimens edges.

Additionally, the acoustic emission (AE) technique was used in order to increase of the accuracy of detect initiation of the peak force values related to the fracture process initiation. 
The AE signal was registered by the piezoelectric sensor mounted on unloaded end of the specimens. The AE phenomena was used as one criterion in calculation of the mode I critical strain energy release rate.

\section{Initiation criteria}

Owing to difficulties in defining the exact instant of crack of crack initiation four definitions for an initiation value of $G_{\text {IC }}$ were used. These include mode I critical strain energy release rate values determined using the load and deflection measured at the point of deviation from linearity in the load-displacement curve (NL), at the maximum load values and at the point which the compliance has increased by $5 \%$. The fourth definition is AE phenomena which was mentioned in previous subsection. The NL $G_{\text {IC }}$ value is typically the lowest of the four initiation values and is recommended for generating delamination failure criteria in durability and damage tolerance analyses of laminated composite structures. The exemplary load-displacement curve obtained during one DCB experiments with depicted initiation points was presented in Fig. 2.



Fig. 2. Exemplary load-displacement curve and energy of AE signal obtained during one of the DCB experiment with determined delamination initiation point definitions.

\section{Calculation methods}

To determine the mode I critical strain energy release rate two data reduction methods were used. These consisted of a Compliance Calibration Method CCM and the Modified Beam Theory MBT, 
The CCM method determines additional parameter $n$ which is the slope of $\ln (C)$ versus $\ln (a)$ curve. Therefore, the critical SERR is calculating as follows:

$$
G_{\mathrm{IC}}=\frac{n P \delta}{2 a b}
$$

MBT method uses correction parameter $\Delta$ which may be determined experimentally by generating a least squares plot of the cube root of compliance $C^{1 / 3}$ as a function of delamination length $a$. The load and displacements corresponding to the visually observed delamination onset on the edge and all the propagation values were used to generate this plot. Mode I interlaminar fracture toughness is expressed following equation:

$$
G_{\mathrm{IC}}=\frac{3 P \delta}{2 b(a+|\Delta|)}
$$

\section{Results and discussion}

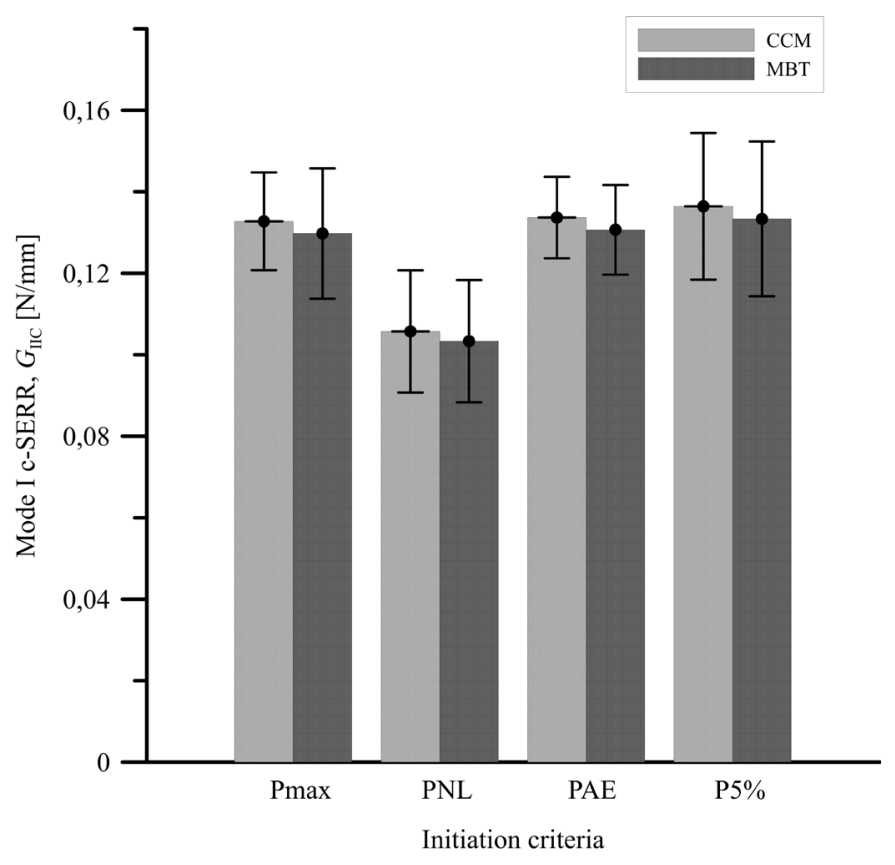

Fig. 3. Mode I c-SERR calculated for different initiation criteria for the specimen with delamination interface $30^{\circ} / 30^{\circ}$ 


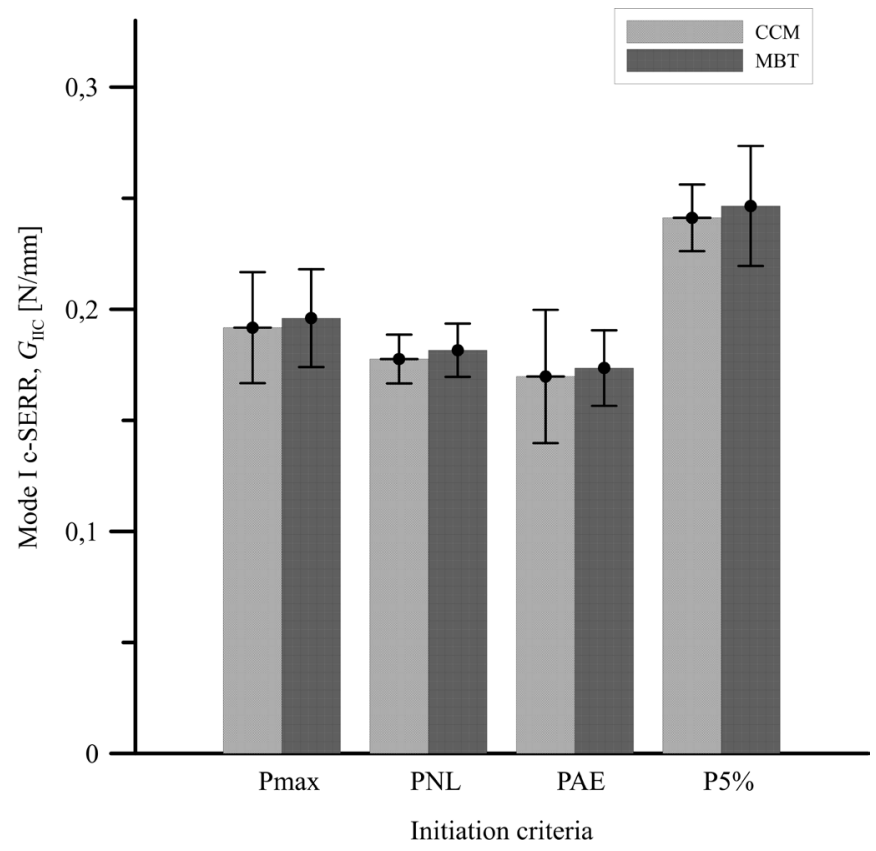

Fig. 4. Mode I c-SERR calculated for different initiation criteria for the specimen with delamination interface $45^{\circ} / 45^{\circ}$

Fig. 3 and Fig. 4 presents the experimentally obtained values of the mode I critical strain energy release rate for specimens with $30^{\circ} / 30^{\circ}$ and $45^{\circ} / 45^{\circ}$ delamination interface calculated for four different criteria. For both specimen, the $G_{\text {IC }}$ reached the greatest values for the $5 \%$ initiation definition. Slightly less value of the c-SERR was obtained for the maximum load point criterium which gave similar values like for the AE and the NL initiation points, whereus, for deviation from linearity definitions the values of the $G_{\mathrm{IC}}$ were the lowest.

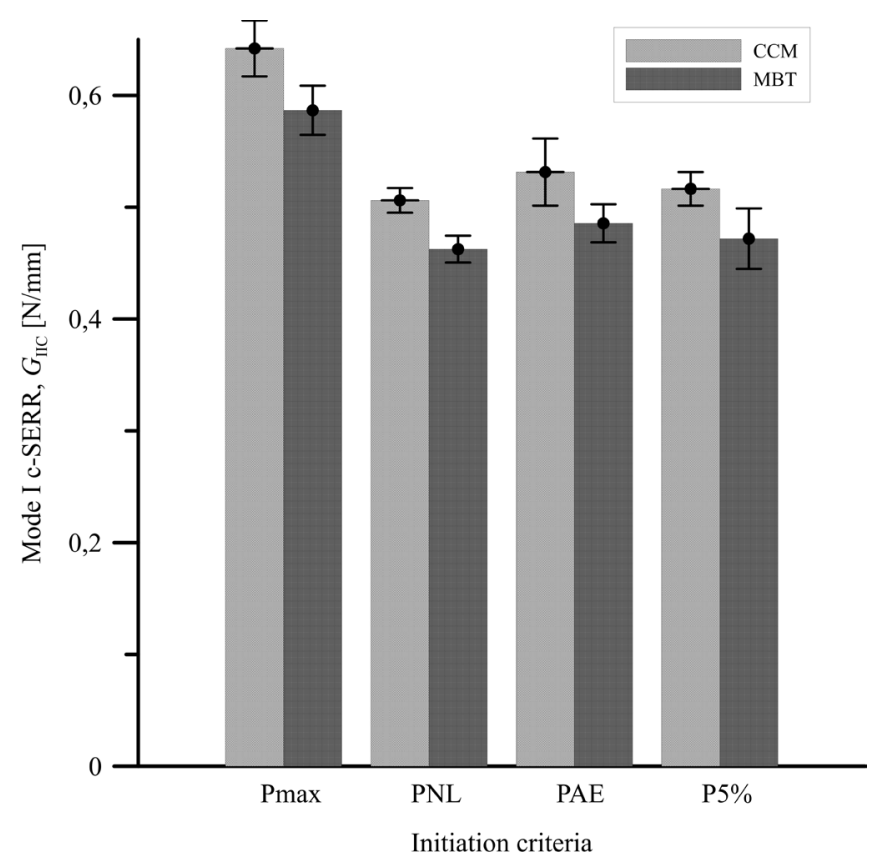

Fig. 5. Mode I c-SERR calculated for different initiation criteria for the specimen exhibiting bending-extension coupling 


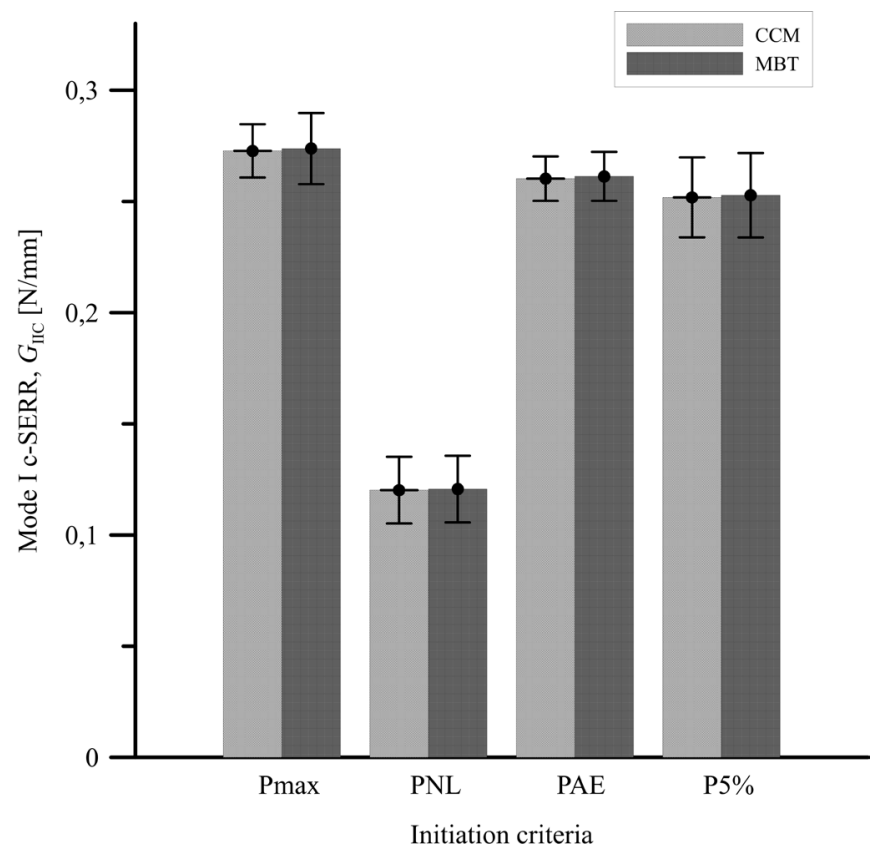

Fig. 6. Mode I c-SERR calculated for different initiation criteria for the specimen exhibiting bending-twisting coupling

Mechanically coupled laminates exhibited different values of the mode I critical strain energy release rate obtained for four different criteria than multidirectional composites. For the bending-extension coupling values of the $G_{\text {IC }}$ were on average level about $0.55 \mathrm{~N} / \mathrm{mm}$ where for bending-twisting coupling this values reached about $0.28 \mathrm{~N} / \mathrm{mm}$. The greatest values of the mode I c-SERR was obtained for the $P_{\max }$ initiation criterium. The results of calculation of the $G_{\text {IC }}$ for the $\mathrm{AE}$ and the $5 \%$ initiation points were alike and equal about $0.5 \mathrm{~N} / \mathrm{mm}$ and $0.25 \mathrm{~N} / \mathrm{mm}$ respectively for the BE and the BT specimens. For both samples, the c-SERR exhibited the lowest values for the deviation from linearity delamination initiation point.

For the CCM and the MBT methods the differences between the results of calculations of the mode I c-SERR for all specimens were minimal.

The experimental values of the mode $G_{\text {IC }}$ obtained for mechanically coupled laminates for different initiation definitions was depicted in Fig. 5 and Fig. 6.

\section{Conclusions}

The critical analysis of the DCB test configuration and direct applicability of the ASTM D5528 Standard in case of the mechanically coupled laminated composite beams was performed. The different initiation criteria was exploited to obtained the mode I critical strain energy release rate. Additionally to calculated the values of $G_{I C}$ the CCM and the MBT methods were used. The results shows that the mode I c-SERR depends on specimen interface, mechanical coupling as well as the adopted initiation point.

\section{Acknowledgments}

The paper was financially supported by the Ministerial Research Project No. DEC2016/21/B/ST8/03160 financed by the Polish National Science Centre. 


\section{References}

[1] Syafigah Nur Azirie Safri et.al, Impact behaviour of hybrid composites for structural applications: A review, Compos. Part. B 133 (2018) 112-121.

[2] ASTM D 5528-01. Standard test method for mode I interlaminar fracture toughness of unidirectional fiber-reinforced polymer matrix composites (2001). https://doi.org/10.1520/d6671_d6671m-13

[3] A. B. Pereira, A. B. de Morais, Mode I interlaminar fracture of carbon/epoxy multidirectional laminates, Comp. Sci. Tech 64 (2004) 2261-70. https://doi.org/10.1016/j.compscitech.2004.03.001

[4] A. B. Pereira, A. B. de Morais,, Mode II interlaminar fracture of glass/epoxy multidirectional laminates, Compos. Part A 35(2) (2004) 265-72.

https://doi.org/10.1016/j.compositesa.2003.09.028

[5] S. Samborski, Numerical analysis of the DCB test configuration applicability to mechanically coupled fiber reinforced laminated composite beams, Compos. Struct 152 (2016) 477-487. https://doi.org/10.1016/j.compstruct.2016.05.060

[6] S. Samborski, Analysis of the end-notched flexure test configuration applicability for mechanically coupled fiber reinforced composite laminaes, Compos. Struct 163 (2017) 342-349. https://doi.org/10.1016/j.compstruct.2016.12.051

[7] S. Samborski, Prediction of delamination front's advancement direction in the CFRP laminates with mechanical couplings subjected to different fracture toughness tests, Compos Struct 202 (2018) 643-650. https://doi.org/10.1016/j.compstruct.2018.03.045

[8] S. Samborski, J. Rzeczkowski, Numerical modeling and experimental testing of the DCB laminated composite beams with mechanical couplings, AIP. Conf. Proc 1922 (2018) 080010-1 - 6. https://doi.org/10.1063/1.5019081

[9] J. Rzeczkowski, S. Samborski, J. Paśnik, Experimental verification on the DCB test configuration applicability to mechanically coupled composite laminates, IOP. Conf. SER.: Mater. Sci. Eng, 416 (2018) 012055. https://doi.org/10.1088/1757-899x/416/1/012055

[10] J. Rzeczkowski, S. Samborski, J. Paśnik, Experimental investigation of mechanically coupled composite specimens in the ENF test configuration., IOP Conf. Ser.: Mater. Sci. Eng. 416, (2018) 012041. https://doi.org/10.1088/1757-899x/416/1/012041

[11] S. Samborski, J. Rzeczkowski, J. Paśnik, Issues of direct application of fracture toughness determination procedures to coupled composite laminates, IOP Conf. Ser.: Mater. Sci. Eng. 416 (2018) 012056. https://doi.org/10.1088/1757-899x/416/1/012056

[12] J. Paśnik, S. Samborski, J. Rzeczkowski, Application of the CZM technique to delamination analysis of coupled laminate beams, IOP Conf. Ser.: Mater. Sci. Eng. 416, (2018) 012075. https://doi.org/10.1088/1757-899x/416/1/012075 